\title{
Detección de peatones con variaciones de forma al caminar con Modelos de Forma Activa
}

\section{Pedestrian's detection with shape variations when walking with Active Shape Models}

\author{
Juan Alberto Antonio \\ Universidad Autónoma del Estado de México, México \\ jaantoniov@uaemex.mx \\ (D) http://orcid.org/0000-0003-3052-3171 \\ Marcelo Romero \\ Universidad Autónoma del Estado de México, México \\ mromeroh@uaemex.mx \\ (D) http://orcid.org/0000-0002-4758-8484
}

Recepción: 27 de marzo de 2019

Aprobación: 11 de febrero de 2020

\section{Resumen}

Se provee un detector de peatones con el algoritmo modelos de forma activa (ASM), con las etapas entrenamiento (PDM) y ajuste (ASM). Con PDM, se marcan 50 landmarks y se extraen los perfiles de grises en la silueta de cada peatón en 137 imágenes (peatón 1 y peatón 2) aplicando los modos de variación (PCA). El aporte de este trabajo es el ajuste y detección de un peatón a pesar de las variaciones. Al final con los resultados evaluados con leave one out en cada imagen de $1080 \times 720$ pixeles y con la métrica del error cuadrático medio (MSE) se obtiene un promedio total de 12.7 pixeles en la distancia de error entre los landmarks originales y los landmarks estimados.

Palabras clave: modelo de formas activas, marcado, ajuste, variaciones de forma.

\begin{abstract}
A pedestrian detector is provided with the algorithm models of active shape (ASM), with the stages: training (PDM) and adjustment (ASM). With PDM, 50 landmarks are marked, and gray profiles are extracted in the silhouette of each pedestrian in 137 images (pedestrian 1 and pedestrian2) applying the variation modes (PCA). The contribution of this work is the adjustment and detection of a pedestrian despite the variations. At the end, the results evaluated with leave one out in each $1080 \times 720$ pixels image and with the mean square error (MSE) metric, a total average of 12.7 pixels is obtained in the error distance between the original landmarks and the estimated landmarks.
\end{abstract}

Keywords: Active Shape Models, marking, adjustment, shape variations.

\section{INTRODUCCIÓN}

La detección de personas es de suma importancia para el estudio en muchas aplicaciones como la seguridad aeroportuaria, sistemas en centros comerciales, escuelas, entre otros (Fang et al., 2017).

La detección de peatones ( $P D$, Pedestrian Detection), como otra disciplina de la detección de personas, consiste en captar sujetos en diferentes poses de pie. Sus desafíos se basan en la gran variabilidad de la apariencia de los peatones debido a la pose, la vestimenta, la oclusión, condiciones de luz, el fondo y, en algunos casos, a las condiciones climáticas y de iluminación (Jordão y Schwartz, 2016; Angonese y Ferreira Rosa, 2017; Kim y Lee, 2013; Halidou et al., 2014; Lakshmi, Faheema y Deodhare, 2016). 
La detección de peatones utilizando cámaras de videovigilancia necesita de métodos que ayuden a capturar sus diferentes posturas al caminar, las cuales pueden ser cambiantes dependiendo de sus características (Dollár et al., 2012) y por las variaciones de su cuerpo.

En la actualidad la detección de peatones se ha realizado a través de diferentes algoritmos, por ejemplo el uso de técnicas deformables como Staticals Shape Models (SSM), Active Contour Models (ACM, Snakes), Active Apearence Models (AAM), etc. (Hill, Thornham y Taylor, 2013; Blake, Curwen y Zisserman, 1993; Enzweiler y Gavrila, 2009; Hilario et al., 2005; Pentland y Horowitz, 1991; Vandenbroucke et al., 1997; Zhang, Bauckhage y Cremers, 2015), los cuales se basan en el ajuste de las formas en las imágenes que contienen objetos por detectar. La problemática que representan estos métodos es la captura de la textura y además requiere una correspondencia y convergencia local mínima ante las variaciones de la forma (Flohr y Gavrila, 2013).

En este artículo se propone el uso del Modelo de Formas Activas (ASM, Active Shape Models), el cual detectará y ajustará mediante landmarks (puntos característicos) escenas de peatones videograbados desde una cámara estática. De acuerdo con esto, la aportación que se pretende es el ajuste del ASM considerando la variabilidad al caminar, i. e., abriendo y cerrando los pies. Esta variabilidad es ajustada mediante una pirámide de resolución (Cootes et al., 1995), la cual disminuye la cantidad de iteraciones necesarias para ajustar el modelo a pesar de las variaciones y los niveles de grises que se puedan encontrar, y que además es un tema poco explorado en el estado del arte (Das Choudhury y Tjahjadi, 2013; Müller y Arens, 2010; Ogawara, Li y Ikeuchi, 2007).

\section{Detección de peatones con el Modelo de Formas Activas}

El estado del arte basado en ASM tiene una literatura amplia relacionada con el ajuste de formas en órganos del cuerpo como manos o rostros (Huysmans, Moens y Van Audekercke, 2005; Razali y Wahab, 2011; Le et al., 2012). Sin embargo, en lo referente a la detección de peatones utilizando el algoritmo ASM, la literatura es limitada debido a que el uso de esta técnica es compleja y requiere un alto consumo de tiempo de cómputo (Cootes et al., 1995); por este motivo, la gran mayoría de las investigaciones realizadas se basan en la detección de personas estáticas (pose de pie sin moverse), y en algunos trabajos se menciona cómo se detectan peatones mientras caminan, lo que permite analizar su cadencia (gait recognition).

Baumberg y Hogg (1994) demuestran cómo se puede dar seguimiento a un cuerpo no rígido en movimiento. Su detector funciona correctamente para poses y vistas aun con dos o tres peatones. La detección del peatón es mediante una silueta elipsoidal entrenada con 40 puntos característicos de la imagen (landmarks); no obstante, no muestran resultados de la efectividad de su propuesta.

Koschan et al. (2003) aplican ASM para detectar cuerpos no rígidos en una secuencia de video mediante una implementación jerárquica en espacios de color $R G B, Y U V$ y $H S I$. Marcan diferentes números de landmarks $(10,14,21$ y 42) y obtienen tres alineaciones de siluetas de una sola persona. Realizan detección de contorno y al final muestran el error normalizado entre los landmarks originales y los landmarks ajustados.

Jung (2008) propone un modelo con base en un exoesqueleto para poder detectar poses humanas. Usan sustracción de fondo y un algoritmo de coincidencia de ASM en el proceso de ajuste. Experimentan con 600 imágenes de personas con 17 landmarks que muestran características de pose. En los resultados obtienen tres componentes principales con un $80.62 \%, 93.3 \%$ y $97.32 \%$ de precisión.

Kim, Lee y Paik, (2009) identifican peatones en su forma de caminar a partir de una secuencia de 122 de personas obtenidas del conjunto de datos HGCD (Sadoghi Yazdi, Fariman y Roohi, 2012). Detectan humanos automáticamente con ASM utilizando 32 landmarks para la silueta de un humano a partir de imágenes de 720 x 480 pixeles. Al final obtienen una efectividad de $90 \%$ en la detección. 
Lee y Choi (2011) detectan peatones utilizando ASM usando imágenes en infrarrojo (IR) y cámaras visibles que permiten seguirlos peatones en entornos degradados y con poca luz. Marcan manualmente 42 landmarks alrededor del cuerpo y al final no muestran resultados en sus experimentaciones.

Ma y Ren (2011) proponen un método basado en ASM para reconocer peatones. No especifican el número de landmarks para entrenar a sus peatones. Las imágenes con resolución de $320 \times 240$ pixeles fueron obtenidos de una cámara $R G B$ omnidireccional. Al final muestran un $94 \%$ de éxito en la detección.

Ide (2013) combina ASM y GrabCut y lo llama segmentación de retroalimentación de forma (ASFSeg), el cual segmenta peatones. Este método compara resultados entre GrabCut y las muestras de ASM y elige la coincidencia para obtener la mejor segmentación. La tasa de error se muestra con foreground $(F G)$ error con $2.32 \%$ y background $(B G)$ error con un $2.15 \%$.

Por su parte, Vasconcelos y Tavares (2015) detectan peatones que caminan en direcciones diversas. Para esto usan el conjunto de datos CASIA Gait Database (Arai y Andrie, 2012) obtenidos de videos en formato MPEG con escenas de peatones en imágenes de $320 \times 240$ pixeles. Entrenan 14 imágenes que representan la silueta del peatón y cada forma se representa por una serie de 113 landmarks, 100 landmarks de la silueta, combinado con 13 landmarks pertenecientes a codos, rodillas y pies. Sus resultados fueron de aproximadamente a un $95 \%$ de las imágenes de un peatón en movimiento en cuatro direcciones.

Por otro lado, en los trabajos relacionados con la detección de transeúntes no mencionan cómo ajustan la apertura completa del compás de las piernas (variación de forma al caminar), ya que muestran una segmentación de apertura de piernas poco representativa. En esta investigación se propone la detección de la variación al caminar con compás abierto o compás cerrado en una imagen en gris que contenga un peatón.

\section{Modelos de forma aCtiva}

Los Modelos de Formas Activas (ASM) es un método flexible que ha sido usado para modelar y representar un amplio rango de objetos (Cootes y Taylor, 1992). En la primera etapa de ASM se realiza el modelo de distribución de puntos (PDM), el cual especifica la forma media del objeto modelado, además de las variaciones que pueda tener; para que el PDM sea robusto es necesario delimitar el contorno de la silueta mediante el marcado con los landmarks (figura 1). Adicionalmente, al modelo de distribución de puntos se añade uno de perfiles de grises basado en la obtención de los niveles de grises en cada landmark y así se podrá obtener en la segunda etapa del algoritmo ASM el ajuste a un nuevo objeto que contenga el perfil de gris más parecido a los niveles adquiridos en el entrenamiento.

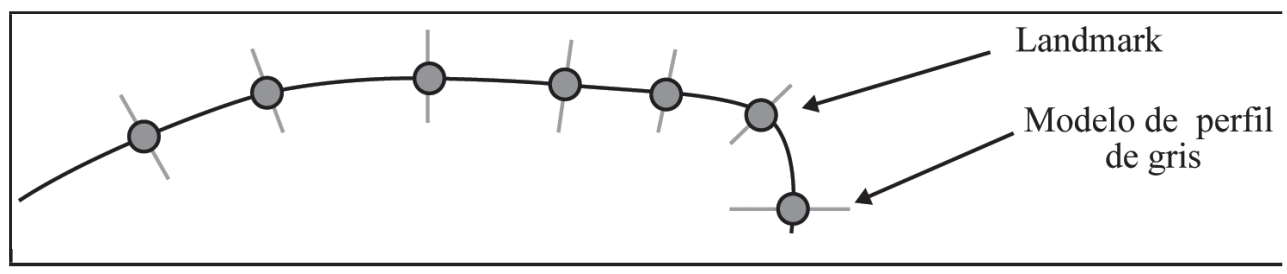

FIGURA 1

Representación del modelado de puntos (landmarks, color verde) y la obtención de los perfiles de grises (línea amarilla) Fuente: Scott, Cootes y Taylor (2003).

A continuación, se muestran las etapas del algoritmo propuesto para la detección de peatones con variaciones al caminar, el cual se basa en el modelo de distribución de puntos de un conjunto de imágenes PDM y el ajuste a una nueva imagen ASM. 


\section{Modelo de distribución de puntos (PDM, Points Distribution Model)}

El modelo de distribución de puntos (PDM) consta de las siguientes etapas:

a) Marcado de landmarks: se obtienen con base en un marcado de puntos estratégicos alrededor de un objeto, el cual pertenece a un conjunto de datos de entrenamiento denotado por la ecuación:

$$
x=\left[x_{1}, \ldots, x_{n}, y_{1}, \ldots, y_{n}\right]^{T}
$$

b) Alineación del conjunto entrenamiento: se alinea el conjunto de entrenamiento mediante una matriz de transformación para buscar la mejor pose de los parámetros de forma y $M$ la matriz de transformación.

$$
X=M(s, \theta)[x]+x
$$

donde:

$$
M=\left[\begin{array}{l}
x_{0} \\
y_{0}
\end{array}\right]=s\left[\begin{array}{rr}
\cos \theta & \operatorname{sen} \theta \\
-\operatorname{sen} \theta & \cos \theta
\end{array}\right]\left[\begin{array}{l}
x_{0} \\
y_{0}
\end{array}\right]+\left[\begin{array}{l}
t_{x} \\
t_{y}
\end{array}\right]
$$

$M(s, \theta)[x]$ alinea una rotación por $\theta$, donde $s$ es el parámetro de escalado y $x$ el conjunto de entrenamiento de imágenes marcadas.

A partir de la ecuación 3 se obtiene la matriz $M$ y posteriormente se genera el nuevo conjunto de entrenamiento alineado $\left(X_{i}\right.$, ecuación 4$)$

$$
X_{i}=M x+(t x, t y)
$$

c) Obtención de los componentes principales (PCA)

- Cálculo de la media de las $m$ formas del conjunto de entrenamiento.

$$
\bar{x}=\frac{1}{m} \sum_{i=1}^{m} X_{i}
$$

- Cálculo de la matriz de covarianza $S$, del conjunto de entrenamiento.

$$
S=\frac{1}{m} \sum_{i=1}^{m}\left(X_{i}-\bar{x}\right)\left(X_{i}-\bar{x}\right)
$$

- $\quad$ Construcción de la matriz donde $\varphi_{j}, j=1 \ldots q$ son los eigenvectores de $S$.

$$
\Phi=\left[\phi_{1}\left|\phi_{2}\right| \ldots \mid \phi_{q}\right]
$$

- $\quad$ Dado $\Phi$ y $x$, donde cada forma pueda aproximarse como:

$$
x_{i} \approx \bar{x}+\Phi b_{i}
$$

donde: 


$$
b_{i}=\Phi^{T}\left(x_{i}-\bar{x}\right)
$$

d) Obtención de los perfiles de grises suavizados: el conjunto de niveles locales de grises (LGL, Local Gray-Level) son usados para capturar el nivel de gris local con su variación observado en cada landmark de la forma PDM (ecuación 10). A continuación se obtiene un modelo estadístico deformable donde $g_{j}, j=1 \ldots n$ es el perfil de grises derivado y normalizado (gráfica 1).

$$
f\left(g_{j, m}\right)=\left(g_{j, m}-\bar{g}_{j}\right)^{T} S_{j}^{-1}\left(g_{j, m}-\bar{g}_{j}\right)
$$

\section{1. Ajuste a una nueva imagen: evaluación del modelo}

\section{1. 1. Obtención de la pirámide de resolución}

Cuando existen problemas con la longitud del perfil de gris, es necesario buscar el perfil más corto, donde la referencia del modelo debe estar cerca de su objetivo en la imagen antes del ajuste del modelo actual.

En caso de que los perfiles sean demasiado largos, la búsqueda se vuelve computacionalmente costosa y los niveles de gris se adhieren a otras estructuras alejadas del objeto de interés haciendo que ASM converja en la forma correcta. Debido a esto, se sugiere un enfoque de resolución múltiple de modo que la imagen tenga desde una baja hasta una alta resolución, generado con un suavizado gaussiano y submuestreo para producir una pirámide de resolución (figura 2). El nivel 0 de la pirámide es la imagen original y el nivel 1 es una imagen con la mitad del número de pixeles a lo largo de cada eje.

Después se utiliza una máscara gaussiana de $5 \times 5$ (se descompone linealmente en 2 circonvoluciones de 1-58-5-1) y luego submuestreando cada pixel del gris correspondiente.

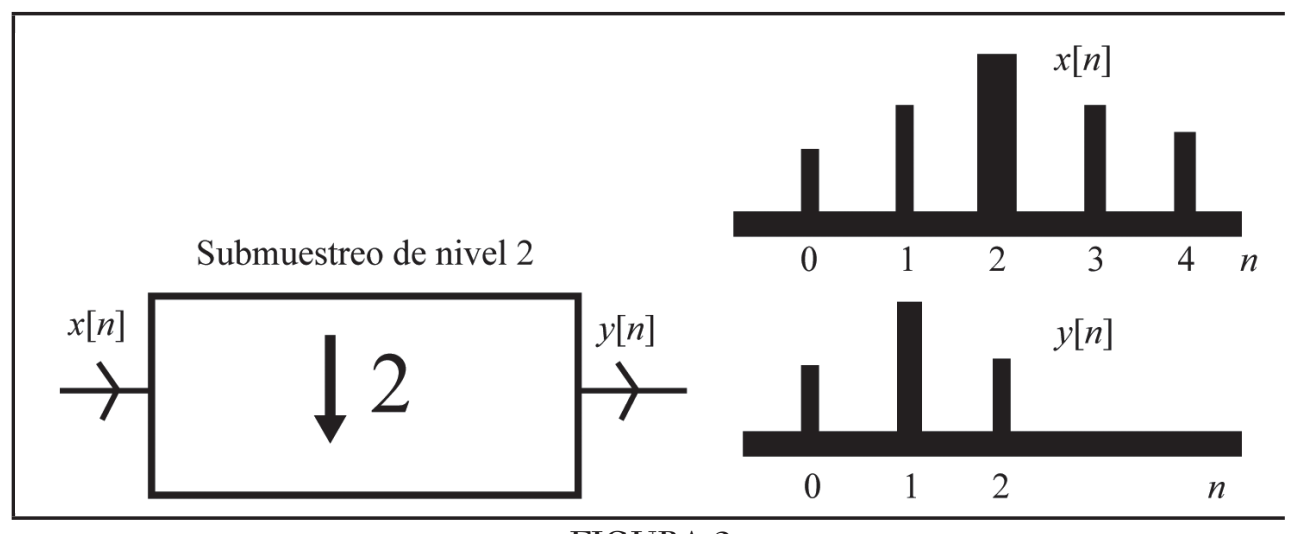

FIGURA 2

Ejemplo de submuestreo en el cambio de resolución en una pirámide de resolución de una imagen Fuente: Scott, Cootes y Taylor (2003).

$$
y[n]=\sum_{n} x[2 n] e^{-i \omega n}
$$

Posterior al ajuste de la nueva imagen con la pirámide resolución se obtiene el ajuste en base a los landmarks y los perfiles de grises:

$$
b_{g}=P_{g}^{T} * y[n](g-\bar{g})
$$




$$
g_{\text {ajustado }}=g+P_{g} b_{g}
$$

$g_{\text {ajustado }}$ representa $g$ desplazado por $m$ muestras a lo largo de la dirección normal del límite correspondiente y con el cual se va midiendo la distancia para encontrar al perfil de gris representativo.
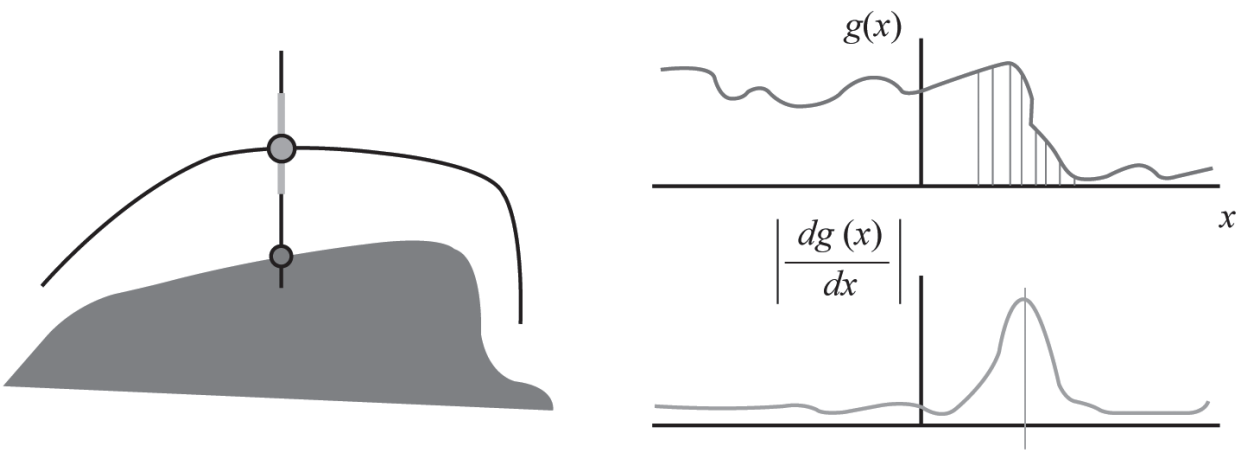

$$
\frac{d g(x)}{d x}=0.5(g(x+1)-g(x-1))
$$

GRÁFICA 1

Búsqueda de los perfiles de grises en un nuevo modelo aplicando el perfil de gris que coincida con el nuevo objeto Nota: Kim y Lee (2013)

De acuerdo con lo expuesto, un modelo estadístico deformable puede ser construido por la asignación de landmarks y la obtención de perfiles de grises. Para poder ajustar a una nueva imagen que no pertenece al conjunto de entrenamiento, se debe encontrar el grupo de parámetros de perfiles grises que mejor coinciden con el modelo de la imagen y que representa los límites del borde que corresponde a la silueta de un peatón.

\section{Proceso experimental en la detección de peatones con variaciones de forma al Caminar}

En esta sección se describe de forma breve la propuesta para la detección de peatones con variaciones de forma al caminar, es decir, compás abierto y cerrado (figura 3). Básicamente, el algoritmo de la figura 3 consiste en dos fases: $a$ ) entrenamiento (PDM) y $b$ ) juste (ASM).

La fase PDM consiste en:

a) Alineamiento de las formas marcadas por los landmarks (ecuaciones 1-4).

b) Análisis de componentes principales (ecuaciones 5-9).

c) Construcción de niveles de grises (ecuación 10).

La fase de ajuste también conocida como ciclo ASM se pude describir como sigue:

d) Transformadas multirresolución aplicadas a las coordenadas de los contornos (ecuación 11).

e) Búsqueda activa (ecuaciones 12-13).

En general, el procedimiento de PDM y ASM propuesto en este trabajo se puede resumir con el Algoritmo 1. Para los fines de este artículo se ajustó el modelo de un peatón con 50 landmarks. 


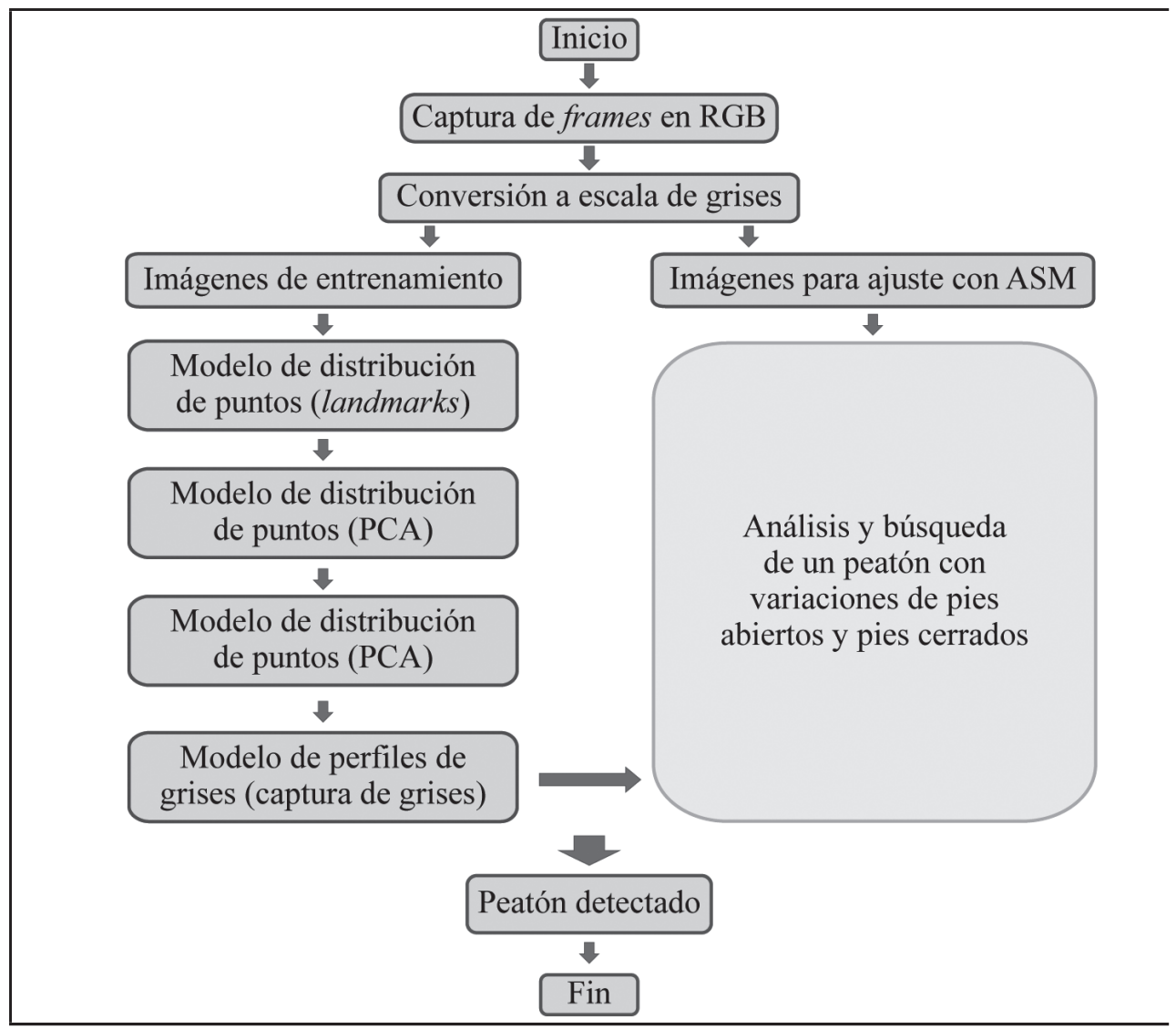

FIGURA 3

Etapas para la detección de un peatón con variaciones de forma con ASM

Fuente: elaboración propia.

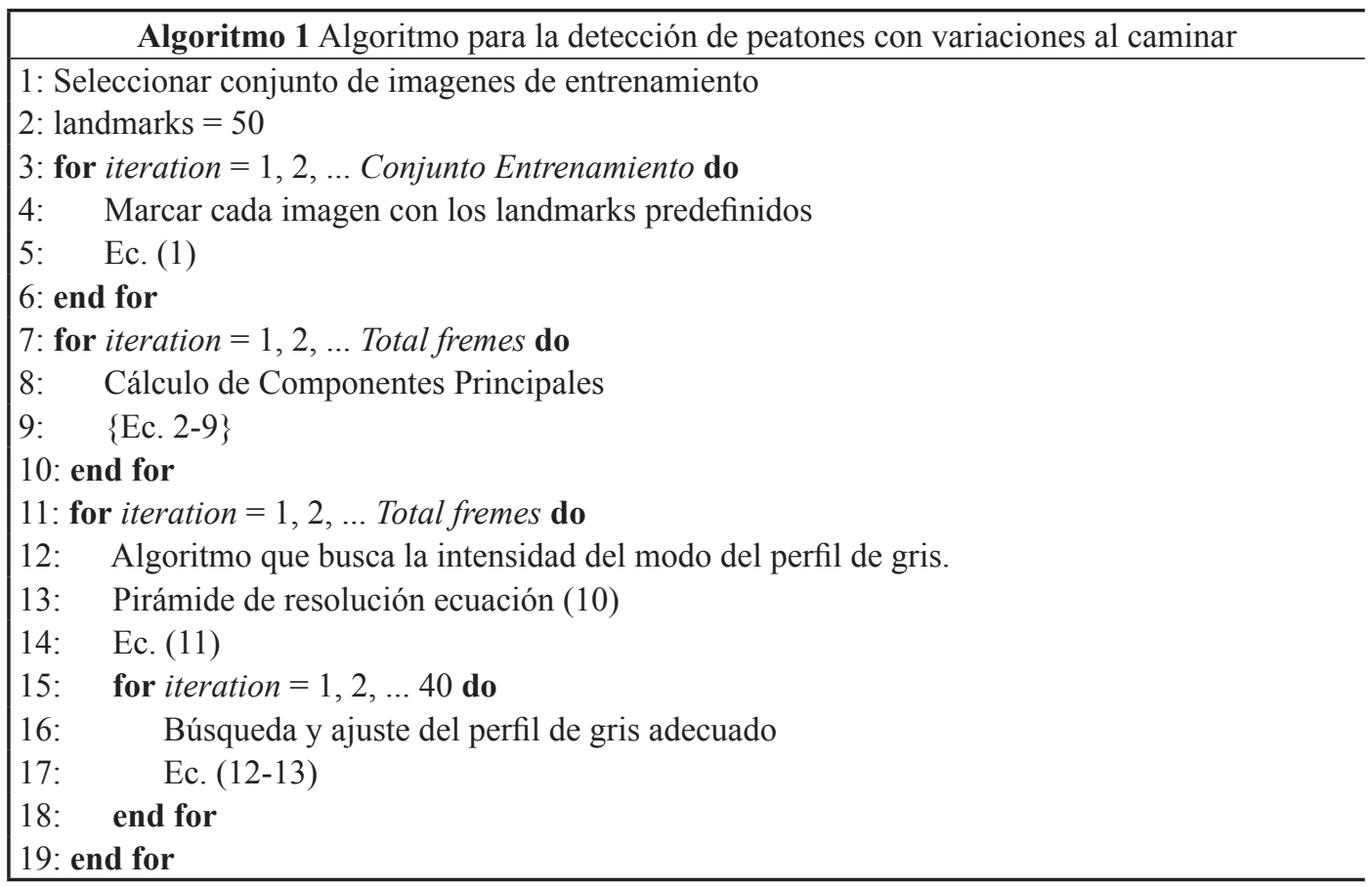

FIGURA 4

Algoritmo 1

Fuente: elaboración propia. 


\section{1. Obtención de los datos experimentales}

La obtención de los datos experimentales fueron a partir de la grabación de un video con escenas de peatones caminando de perfil para así poder obtener la apertura y cierre de piernas. El lugar donde se realizó la grabación fue en un pasillo de $4.50 \mathrm{~m}$ de largo $\times 3.80 \mathrm{~m}$ de ancho en el interior del edificio de posgrado de la Facultad de Ingeniería de la Universidad Autonóma del Estado de México.

Se usó una cámara fija $R G B$ FaceTime $H D$ de 720 p, la cual se localizaba a una distancia aproximada de 1.47 $\mathrm{m}$ de la zona donde caminaron los peatones. Para grabar a los peatones, se tomaron en cuenta varios factores tal como la cadencia de los pasos y la velocidad para poder obtener frames legibles y evitar imágenes borrosas que pudieran tener resultados de detección poco satisfactorios.

Todos los videos obtenidos se almacenaron en formato .mov con una resolución de $1080 \times 720$ pixeles y posteriormente se guardó en formato PNG. Los archivos de imagen PNG no pierden información al cambiarlos a escala de grises, lo cual es útil para ser usado por el algoritmo ASM. La tabla 1 muestra los conjuntos de entrenamiento necesarios en las escenas para el peatón 1 y peatón 2, donde la cantidad de frames se estableció en dos categorías para cada conjunto: $a$ ) pies abiertos y $b$ ) pies cerrados.

TABLA 1

Extracción de imágenes experimentales en peatón 1 y peatón 2

\begin{tabular}{|l|cc|ccc}
\hline \multirow{2}{*}{ Cantidad de frames } & \multicolumn{2}{|c|}{ Peatón 1 } & \multicolumn{2}{c}{ Peatón 2 } \\
\cline { 2 - 6 } & Pies cerrados & Pies abiertos & Pies cerrados & Pies abiertos \\
\hline $\begin{array}{l}\text { Frames individuales } \\
\text { Frames totales }\end{array}$ & 25 & 39 & 44 & 15 \\
\hline
\end{tabular}

Fuente: elaboración propia.

\section{2. Modelado de distribución de puntos}

En la etapa de modelado de distribución de puntos se propuso un marcado de 50 landmarks alrededor del contorno del peatón (Godil, 2007) tomando en cuenta algunos puntos antropométricos según el modelo CAESAR (Ressler, 2001). De este modelo se pueden identificar los puntos específicos que corresponden a ciertas extremidades o partes del cuerpo humano, ya sea en modelos 2D o en 3D. En la figura 5, se muestran las imágenes de entrenamiento y su marcado ( 50 landmarks) correspondiente a los peatones con pies cerrados y con pies abiertos en las escenas de peatón 1 y peatón 2 para ser usados en las etapas de PDM (sección 4).

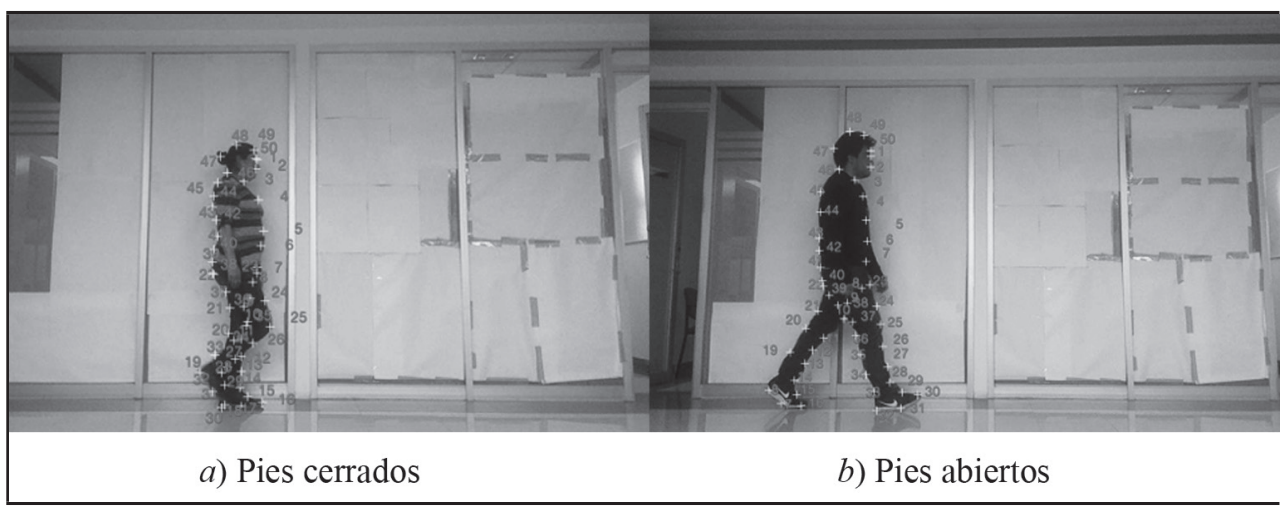

FIGURA 5

Etapas para la detección de un peatón con variaciones de forma con ASM Fuente: elaboración propia. 
Posteriormente, se busca la alineación del conjunto de entrenamiento (gráfica 2), y después se origina la forma media de todo el conjunto de entrenamiento (gráfica 3). Para la captura de perfiles de grises se utilizó una recta perpendicular a cada landmark de 40 pixeles de ancho (20 pixeles hacia arriba y 20 pixeles hacia abajo) y así utilizarlos en la búsqueda activa de grises en cada imagen del conjunto de entrenamiento. Se definieron como criterio de parada 40 iteraciones en la búsqueda del ajuste al mejor modelo de variación que se asemeje para poder segmentar la silueta del peatón tomando en cuenta las variaciones en el movimiento de las piernas al caminar.

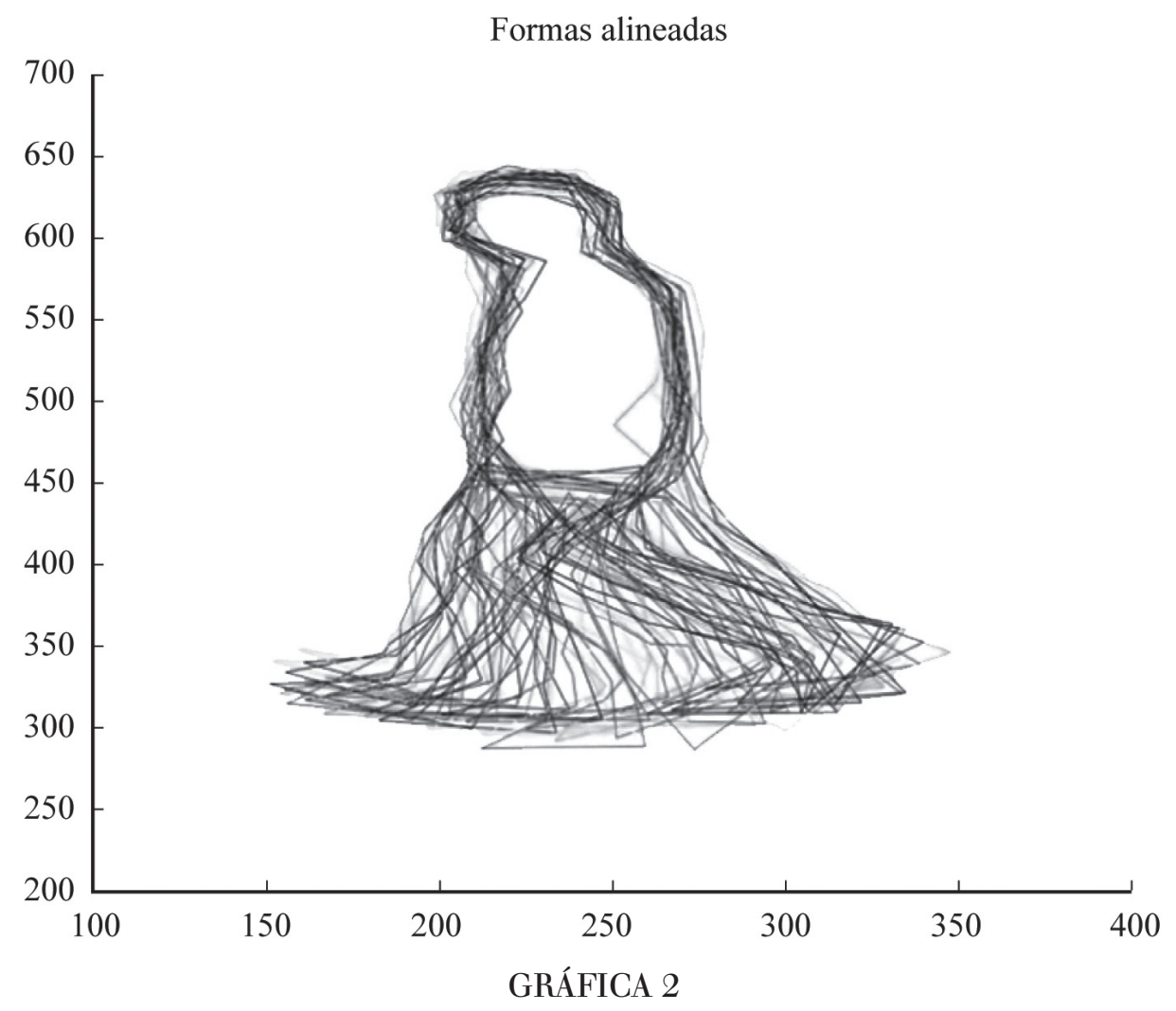

Representación de las formas alineadas en un peatón con variaciones de pies abiertos Nota: elaboración propia.

La gráfica 3 muestra las formas medias del peatón 1 y peatón 2 con pies abiertos y pies cerrados con la técnica PCA.

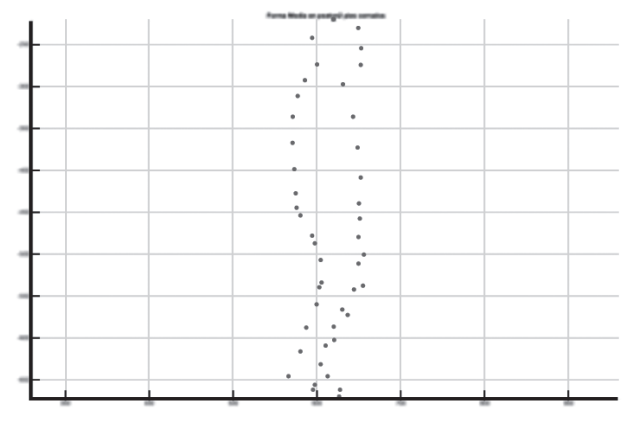

a) peatón 2 , pies cerrados

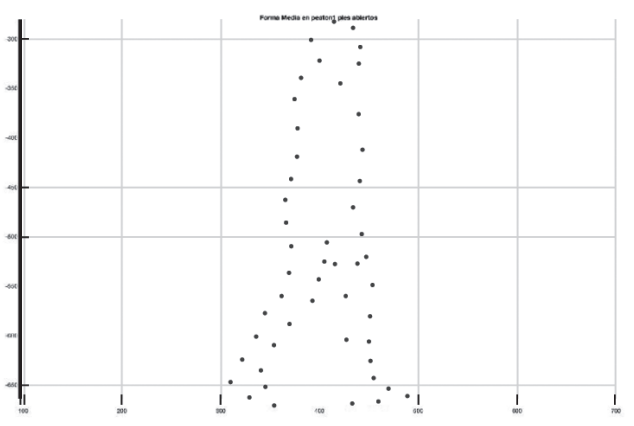

b) peatón 1 , pies abiertos

GRÁFICA 3

Representación de las formas medias en $a$ ) y $b$ )

Nota: elaboración propia. 


\section{Evaluación de Resultados}

El algoritmo ASM fue desarrollado en MATLAB R2016b con un procesador a $2.3 \mathrm{GHz}$ Intel Core i7 y una memoria RAM de $16 \mathrm{~GB}$, y su ejecución ayudó a identificar y segmentar automáticamente el contorno de un peatón compuesto por 50 landmarks para posteriormente realizar el ajuste de la forma.

\section{1. Métrica de evaluación}

Como procedimiento de evaluación se utilizó la validación cruzada, en particular la técnica leave one out (Vehtari, Gelman y Gabry, 2017), la cual sirvió para estimar las imágenes (test) del conjunto de entrenamiento y dicha estimación fue medida con el método del error cuadrático medio (MSE). El procedimiento consistió en calcular la distancia entre los landmarks originales del marcado y los landmarks resultados del ajuste en la imagen de la prueba.

Los resultados de la búsqueda de ajuste con ASM en el peatón 1 (piernas abiertas) y peatón 2 (piernas cerradas) se muestran en la figura 6, en la cual se observa el marcado original (puntos amarillos) y el obtenido por el ajuste del ASM (puntos rojos). La mejor detección se realizó en el ajuste con piernas cerradas, donde la distancia de los puntos rojos está muy cercana al marcado original (puntos amarillos). En la imagen que muestra piernas abiertas (figura 6b) hay una leve separación de los puntos rojos con los puntos amarillos en los pies del peatón 1, lo que significa que en piernas abiertas el modelo ASM no se desempeña de manera óptima debido a la variación de la forma.

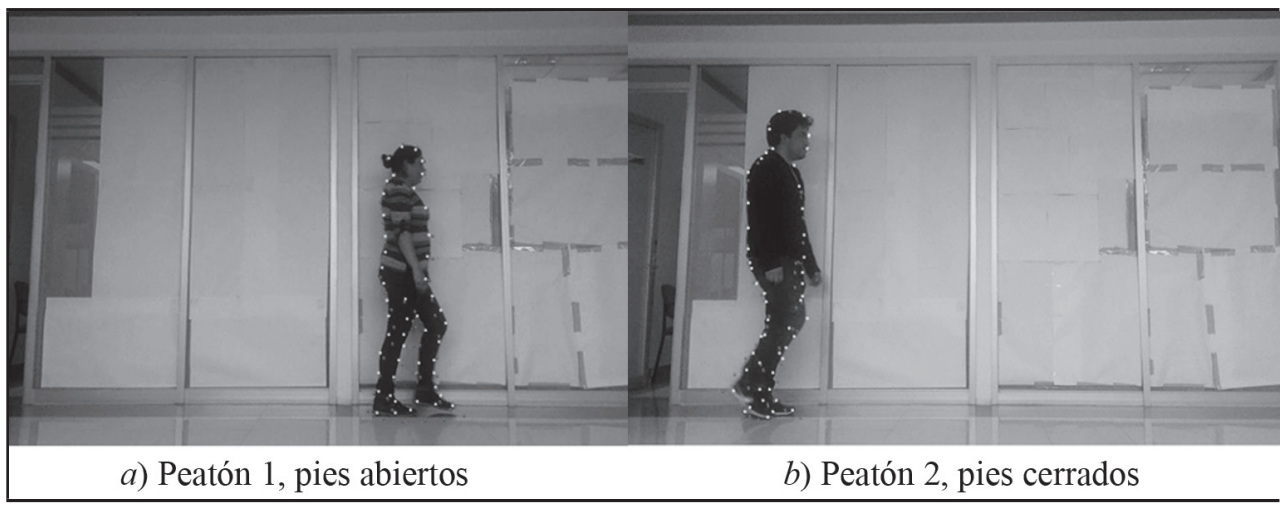

FIGURA 6

Representación del marcado original (puntos amarillos) y el marcado resultante del ajuste (puntos rojos)

Fuente: elaboración propia.

\section{2. Representación de los resultados obtenidos con ASM en los conjuntos de imágenes de peatón 1 y peatón 2}

A continuación se muestran los resultados obtenidos (gráfica 4) con el procedimiento de validación cruzada y la técnica de leave one out cuando se usó como criterio de medida el MSE de la distancia entre el marcado original y el resultado de los landmarks ajustados en cada uno de los conjuntos de imágenes experimentales (peatón 1 y peatón 2 con piernas abiertas y piernas cerradas, respectivamente).

La gráfica 4 muestra el MSE del ajuste del ASM con los 25 frames que contienen la escena de peatón 1 (con pies abiertos). Se observa que el peor resultado en el cálculo de la distancia con MSE entre los landmarks originales y los landmarks ajustados se obtiene con el frame 9 a una distancia de 150 pixeles, mientras que el ajuste más equilibrado se encuentra en el peatón 1, donde se observa que el frame 4 tuvo un resultado de 
10 pixeles. El resultado promedio obtenido en la mayoría de los frames es representado por una línea roja donde se observa un MSE de distancia de 30 pixeles, por lo que se demuestra que el algoritmo ajustó las piernas abiertas en la mayoría de los frames a pesar de las variaciones por la apertura de las mismas.

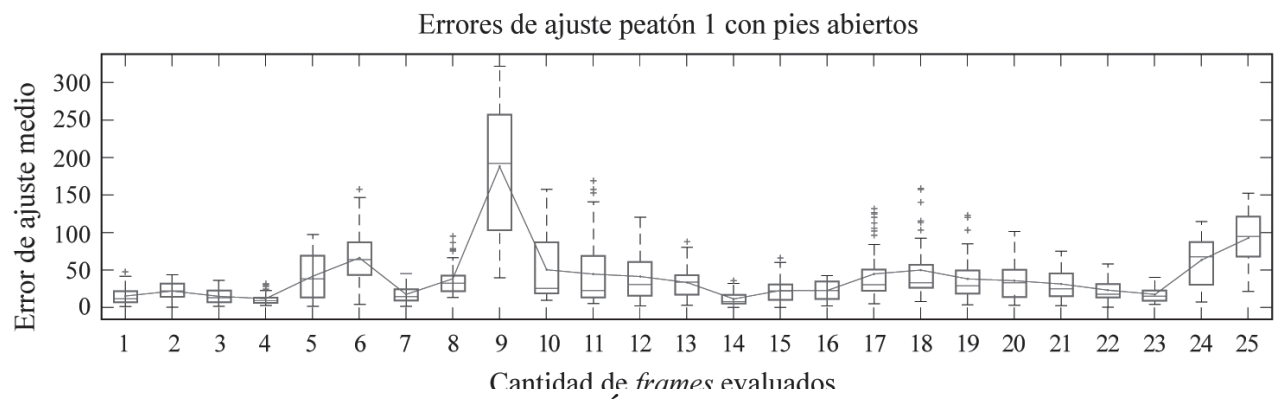

GRÁFICA 4

Representación gráfica de diagrama de caja, donde se muestra la evaluación promedio del

MSE en cada uno de los 25 frames del peatón 1 con pies cerrados

Nota: elaboración propia.

Por su parte, la gráfica 5 corresponde al conjunto de 39 imágenes experimentales del peatón 1 con pies cerrados y muestra mediante una línea roja que la distancia promedio de MSE es de 50 pixeles entre los landmarks originales y los landmarks resultado del ajuste. En algunos frames como 4, 12, 34, 35 y 37 se aprecia que la distancia de MSE es cercana a 0 pixeles demostrando en este caso que la detección del peatón 1 con pies cerrados contiene menos errores de ajuste que la detección con pies abiertos.

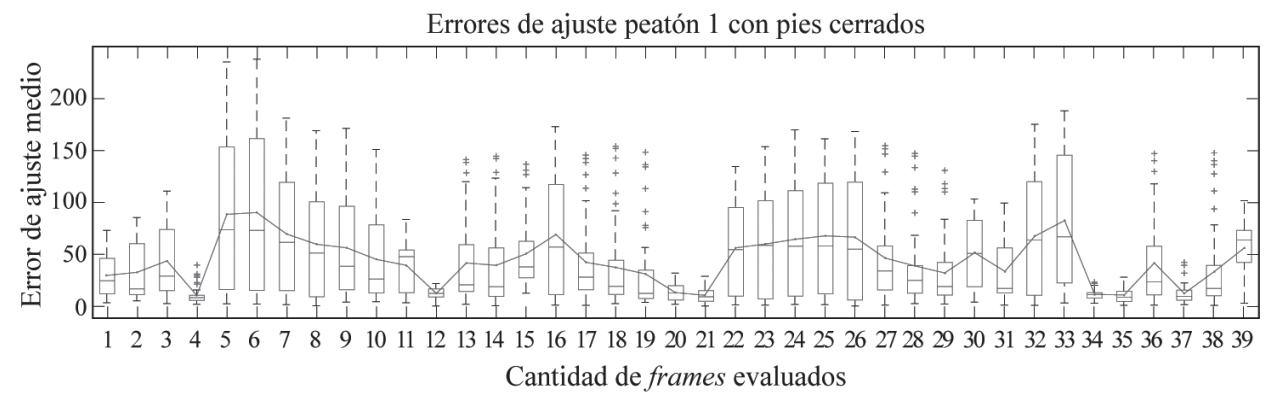

GRÁFICA 5

Representación gráfica de diagrama de caja, donde se muestra la evaluación promedio del MSE en cada uno de los 39 frames del peatón 1 con pies cerrados Nota: elaboración propia.

La gráfica 6 corresponde a la evaluación de 15 frames del peatón 2 con pies abiertos. Muestra mediante la línea roja una distancia de MSE de 20 pixeles entre los landmarks originales contra los landmarks resultado del ajuste. En esta figura se evidencia la efectividad del método ASM, propuesto en este trabajo, en el peatón 2 con pies abiertos.

La gráfica 7 exhibe la evaluación de los 44 frames correspondientes al peatón 2 con pies cerrados. La línea roja señala el promedio de la distancia MSE, la cual es aproximada a 70 pixeles de separación entre los landmarks originales y los landmarks resultado del ajuste en 34 frames. En los frames 1, 4, 19, 20, 21, 23, 24, 25, 26, 27 y 28, del mismo conjunto, la distancia MSE es aproximadamente de 5 pixeles, siendo los frames con mejores ajustes. En otras palabras, en la mayoría de estos ajustes no se obtuvieron resultados satisfactorios; no obstante, en aquellos donde se tuvieron mejores resultados el ajuste fue bastante efectivo (distancia de MSE de 5 pixeles). 


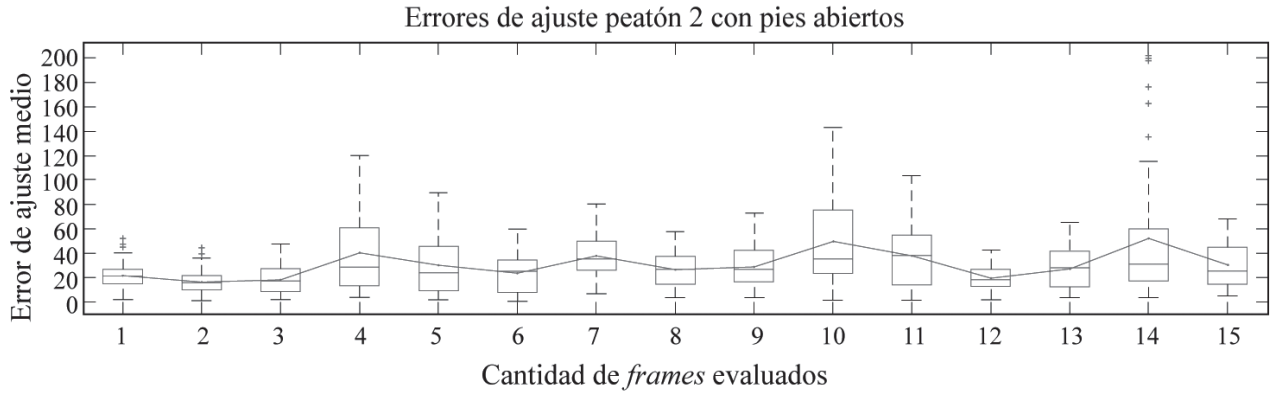

GRÁFICA 6

Representación gráfica de diagrama de caja, donde se muestra la evaluación promedio del MSE en cada uno de los 15 frames del peatón 2 con pies abiertos Nota: elaboración propia.

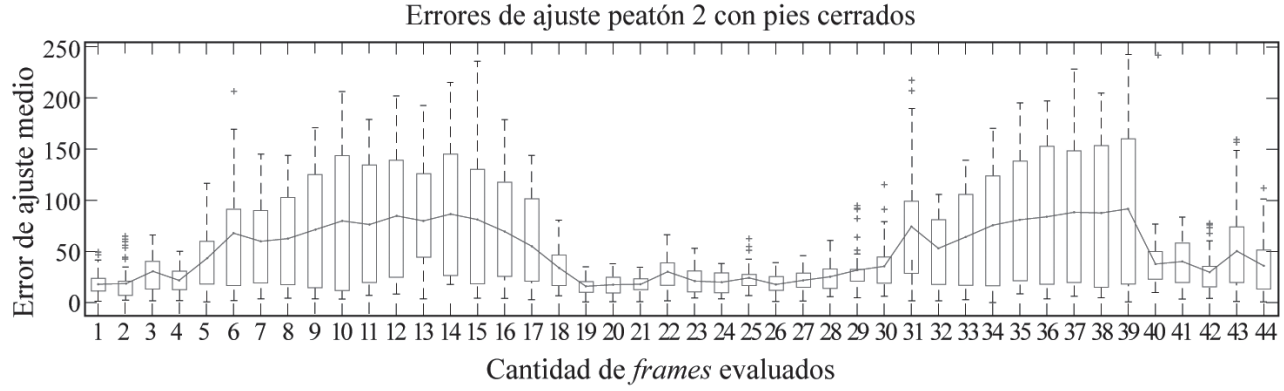

GRÁFICA 7

Representación gráfica de diagrama de caja, donde se muestra la evaluación promedio del MSE en cada uno de los 15 frames del peatón 2 con pies abiertos Nota: elaboración propia.

La tabla 2 muestra el promedio general del cálculo de la distancia MSE entre el marcado original y el marcado ajustado, los cuales fueron evaluados y mostrados en los diagramas de caja en las escenas de peatón 1 y peatón 2 con pies cerrados y pies abiertos. Se puede observar que el peatón 1 con pies cerrados y un conjunto de 39 frames tiene un promedio total de 41.6 pixeles del cálculo de la distancia MSE y con pies abiertos y un conjunto de 25 frames tuvo un promedio total de 12.7 pixeles en el cálculo de la distancia MSE. Por otra parte, el peatón 2 con pies abiertos y un conjunto de 15 frames tuvo un promedio total de 30.4 pixeles en el cálculo de la distancia MSE y con piernas cerradas y un conjunto de 44 frames tuvo un promedio total en el cálculo de la distancia MSE de 50.5 pixeles. Al finalizar estos resultados se puede decir que en algunos resultados la distancia resultado del ajuste (puntos rojos) parece estar muy separada de los landmarks originales resultado del marcado de entrenamiento (puntos amarillos), pero un factor a tomar en cuenta es la resolución de los frames evaluados, que en este caso fue de $1080 \times 720$ pixeles, con lo cual se puede concluir que ASM ajusta de manera correcta a pesar de las variaciones.

\section{TABLA 2}

Promedio total del error MSE de ajuste en peatón 1 y peatón 2 en cada conjunto de frames, ya sea con pies cerrados o con pies abiertos

\begin{tabular}{|lcccc}
\hline \multicolumn{2}{c}{ Peatón 1 } & \multicolumn{2}{c}{ Peatón 2 } \\
\hline Pose & Número de frames & Error medio de ajuste total & Número de frames & Error medio de ajuste total \\
Pies abiertos & 25 & 41.6 & 15 & 30.4 \\
& Número de frames & Error medio de ajuste total & Número de frames & Error medio de ajuste total \\
Pies cerrados & 39 & 12.7 & 44 & 50.5 \\
\hline
\end{tabular}




\section{Conclusiones}

En este artículo se propuso la detección de peatones con variaciones de forma al caminar usando el algoritmo ASM. Se evaluaron dos escenas denominadas peatón 1 y peatón 2, de los cuales se obtuvieron dos conjuntos de frames para cada escena (pies abiertos y pies cerrados). Se entrenó a cada conjunto de imágenes mediante el modelado de distribución de puntos con 50 landmarks alrededor del contorno de la silueta de cada peatón y en una etapa posterior se aplicó el ajuste de forma. Para poder evaluar el desempeño del ajuste se utilizó una validación cruzada, con la técnica leave one out, la cual sirvió para estimar las imágenes del conjunto de entrenamiento midiendo la distancia con el método del error cuadrático medio (MSE) entre los landmarks originales y los landmarks resultados del ajuste. Al final se muestran los mejores resultados dando un promedio total de 12.7 pixeles en la distancia MSE entre los landmarks del marcado original y los landmarks resultado del ajuste en el peatón 1 con pies cerrados, y un promedio total de 30.4 pixeles en la distancia MSE entre los landmarks del marcado original y los landmarks resultado del ajuste en el peatón 1 con pies abiertos.

\section{AnÁlisis PRospectivo}

Dado que se necesitan medidas de seguridad en los sistemas de videovigilancia, es necesario utilizar videos que capturen imágenes en vivo y que se puedan obtener de los centros de control, comando, comunicación, cómputo y calidad (C5) de cualquier entidad gubernamental de México. Con ello, se pueda entrenar y detectar peatones con variaciones al caminar con el algoritmo (ASM) y combinándolo con la fortaleza de otro algoritmo pueda disminuir los problemas de oclusión, iluminación y fondos difíciles. Se espera a futuro que los centros de control (C5) implanten el detector para ubicar peatones sospechosos, sobre todo porque los detectores de peatones modernos ubican y representan gráficamente la detección mediante un rectángulo (bounding box) y no mediante un modelo que ajuste la silueta.

\section{Agradecimientos}

Se agradecen los comentarios de los árbitros de la revista que mejoraron sustancialmente el contenido del artículo.

\section{REFERENCIAS}

Angonese, A. T., \& Ferreira Rosa, P. F. (2017). Multiple people detection and identification system integrated with a dynamic simultaneous localization and mapping system for an autonomous mobile robotic platform. ICMT 2017-6th International Conference on Military Technologies, 779-786. https://doi.org/10.1109/ MILTECHS.2017.7988861

Arai, K., \& Andrie, R. (2012). Gait recognition method based on wavelet transformation and its evaluation with Chinese Academy of Sciences (CASIA) gait database as a human gait recognition dataset. Proceedings of the 9th International Conference on Information Technology, ITNG 2012. https://doi.org/10.1109/ ITNG.2012.164

Baumberg, A. M., \& Hogg, D. C. (1994). An efficient method for contour tracking using active shape models. Proceedings of 1994 IEEE Workshop on Motion of Non-rigid and Articulated Objects. https://doi. org/10.1109/MNRAO.1994.346236

Blake, A., Curwen, R. y Zisserman, A. (1993). A framework for spatiotemporal control in the tracking of visual contours. International Journal of Computer Vision. https://doi.org/10.1007/BF01469225 
Cootes, T. F., \& Taylor, C. J. (1992). Active Shape Models-'Smart Snakes'. BMVC92. https://doi.org/10.1007/9781-4471-3201-1_28

Cootes, T. F., Taylor, C. J., Cooper, D. H., \& Graham, J. (1995). Active shape models-their training and application. Computer Vision and Image Understanding. https://doi.org/10.1006/cviu.1995.1004

Das Choudhury, S., \& Tjahjadi, T. (2013). Gait recognition based on shape and motion analysis of silhouette contours. Computer Vision and Image Understanding. https://doi.org/10.1016/j.cviu.2013.08.003

Dollár, P., Wojek, C., Schiele, B., \& Perona, P. (2012). Pedestrian detection: An evaluation of the state of the art. IEEE Transactions on Pattern Analysis and Machine Intelligence. https://doi.org/10.1109/ TPAMI.2011.155

Enzweiler, M., \& Gavrila, D. M. (2009). Monocular pedestrian detection: Survey and experiments. IEEE Transactions on Pattern Analysis and Machine Intelligence. https://doi.org/10.1109/TPAMI.2008.260

Fang, F., Qian, K., Zhou, B., \& Ma, X. (2017). Real-Time RGB-D based People Detection and Tracking for Mobile. Proceedings of 2017 IEEE International Conference on Mechatronics and Automation, 1937-1941.

Flohr, F., \& Gavrila, D. (2013). PedCut: An iterative framework for pedestrian segmentation combining shape models and multiple data cues. Procedings of the British Machine Vision Conference 2013. https://doi. org/10.5244/C.27.66

Godil, A. (2007). Advanced human body and head shape representation and analysis. Digital Human Modeling, 92-100.

Halidou, A., You, X., Hamidine, M., Etoundi, R. A., Diakite, L. H., \& Souleimanou. (2014). Fast pedestrian detection based on region of interest and multi-block local binary pattern descriptors. Computers and Electrical Engineering. https://doi.org/10.1016/j.compeleceng.2014.10.003

Hilario, C., Collado, J. M., Armingol, J. M., \& De La Escalera, A. (2005). Pedestrian detection for intelligent vehicles based on active contour models and stereo vision. Lecture Notes in Computer Science (including subseries Lecture Notes in Artificial Intelligence and Lecture Notes in Bioinformatics). https://doi. org/10.1007/11556985_70

Hill, A., Thornham, A., \& Taylor, C. J. (2013). Model-Based Interpretation of 3D Medical Images. https://doi. org/10.5244/c.7.34

Huysmans, T., Moens, P., \& Van Audekercke, R. (2005). An active shape model for the reconstruction of scoliotic deformities from back shape data. Clinical Biomechanics. https://doi.org/10.1016/j.clinbiomech.2004.06.015

Ide, I. (2013). Segmentation of Human Instances Using Grab-cut and Active Shape Model Feedback. Computer Science, 11-14.

Jordão, A. y Schwartz, W. R. (2016). The Good, The Fast and The Better Pedestrian Detector. Universidade Federal de Minas Gerais-Departamento de Ciência da Computação, 1, 1-51. Retrieved from http://gibis.unifesp. $\mathrm{br} /$ sibgrapi 16/eproceedings/wtd/19.pdf

Jung, C. J. (2008). Human Pose Estimation ASM. Retrieved from scholar.waset.org/1999.7/12456

Kim, D., Lee, S., \& Paik, J. (2009). Active shape model-based gait recognition using infrared images. Communications in Computer and Information Science, 61(4), 275-281. https://doi.org/10.1007/978-3-64210546-3_33

Kim, D. S., \& Lee, K. H. (2013). Segment-based region of interest generation for pedestrian detection in far-infrared images. Infrared Physics \& Technology. https://doi.org/10.1016/j.infrared.2013.08.001 
Koschan, A., Kang, S., Paik, J., Abidi, B., \& Abidi, M. (2003). Color active shape models for tracking non-rigid objects. Pattern Recognition Letters. https://doi.org/10.1016/S0167-8655(02)00330-6

Lakshmi, A., Faheema, A. G. J., \& Deodhare, D. (2016). Pedestrian detection in thermal images: An automated scale based region extraction with curvelet space validation. Infrared Physics \& Technology. https://doi. org/10.1016/j.infrared.2016.03.012

Le, V., Brandt, J., Lin, Z., Bourdev, L., \& Huang, T. S. (2012). Interactive facial feature localization. Lecture Notes in Computer Science (including subseries Lecture Notes in Artificial Intelligence and Lecture Notes in Bioinformatics). https://doi.org/10.1007/978-3-642-33712-3_49

Lee, D., \& Choi, S. (2011). Multisensor fusion-Based object detection and tracking using Active Shape Model. 2011 6th International Conference on Digital Information Management 2011, 108-114. https://doi. org/10.1109/ICDIM.2011.6093321

Ma, J., \& Ren, F. (2011). Detect and track the dynamic deformation human body with the active shape model modified by motion vectors. 2011 IEEE International Conference on Cloud Computing and Intelligence Systems, 587-591. https://doi.org/10.1109/CCIS.2011.6045137

Müller, J., \& Arens, M. (2010). Human pose estimation with Implicit Shape Models. ARTEMIS'10-Proceedings of the 1st ACM Workshop on Analysis and Retrieval of Tracked Events and Motion in Imagery Streams, Co-located with ACM Multimedia 2010. https://doi.org/10.1145/1877868.1877873

Ogawara, K., Li, X., \& Ikeuchi, K. (2007). Marker-less human motion estimation using articulated deformable model. Proceedings. IEEE International Conference on Robotics and Automation. https://doi.org/10.1109/ ROBOT.2007.363763

Pentland, A., \& Horowitz, B. (1991). Recovery of Non-Rigid Motion and Structure. IEEE Transactions on Pattern Analysis and Machine Intelligence. https://doi.org/10.1109/34.85661

Razali, N., \& Wahab, A. (2011). 2D Affective Space Model (ASM) for detecting autistic children. Proceedings of the International Symposium on Consumer Electronics. https://doi.org/10.1109/ISCE.2011.5973888

Ressler, S. (2001). A Web-based 3D Glossary for Anthropometric Landmarks. Proceedings of HCI International, 1, 1-5.

Sadoghi Yazdi, H., Fariman, H. J., \& Roohi, J. (2012). Gait recognition based on invariant leg classification using a neuro-fuzzy algorithm as the fusion method. ISRN Artificial Intelligence. https://doi. org/10.5402/2012/289721

Scott, I. M., Cootes, T. F., \&Taylor, C. J. (2003). Improving appearance model matching using local image structure. Lecture Notes in Computer Science (including subseries Lecture Notes in Artificial Intelligence and Lecture Notes in Bioinformatics). https://doi.org/10.1007/978-3-540-45087-0_22

Vandenbroucke, N., Macaire, L., Vieren, C., \& Postaire, J. G. (1997). Contribution of a color classification to soccer players tracking with snakes. Proceedings of the IEEE International Conference on Systems, Man and Cybernetics. https://doi.org/10.1109/icsmc.1997.633237

Vasconcelos, M. J. M., \& Tavares, J. M. R. S. (2015). Human motion segmentation using active shape models. Lecture Notes in Computational Vision and Biomechanics. https://doi.org/10.1007/978-3-319-15799-3_18

Vehtari, A., Gelman, A., \& Gabry, J. (2017).Practical Bayesian model evaluation usingleave-one-outcross-validation and WAIC. Statistics and Computing. https://doi.org/10.1007/s11222-016-9696-4

Zhang, S., Bauckhage, C., \& Cremers, A. B. (2015). Efficient pedestrian detection via rectangular features based on a statistical shape model. IEEE Transactions on Intelligent Transportation Systems. https://doi. org/10.1109/TITS.2014.2341042 